\title{
Une stratégie de renforcement des petits centres ruraux
}

\author{
Clermont Dugas \\ Universit du Qu bec Rimouski
}

\section{Introduction}

D'importantes disparités socio-économiques et physicospatiales caractérisent le tissu de peuplement québécois. Elles se manifestent par des déséquilibres affectant la répartition des villes, des services, des emplois et des niveaux de revenus. Elles se traduisent aussi par la présence de zones de marginalité socio-économique dans de nombreuses parties du territoire, et particulièrement en milieu rural. Elles sont également à l'origine d'importants déplacements de population, qui contribuent à l'anémie de certains espaces et à des problèmes d'encombrement et de congestion dans d'autres.

Ces disparités résultent d'une multiplicité de facteurs parmi lesquels la structure de peuplement occupe une place importante. L'emplacement des localités les unes par rapport aux autres et en regard des diverses composantes de l'infrastructure de communications, leurs différences de taille démographique, leurs éléments de dépendance et de complémentarité sont autant de faits qui contribuent à la dynamique spatiale et à celle de l'économie.

À l'échelon inférieur de la hiérarchie urbaine, les petits centres de services contribuent à la structuration du monde rural. Bien que dotés d'une infrastructure de services relativement modeste, ils jouent un rôle important dans la vie de relations et l'économie de leur région d'insertion. Le but principal du présent article est de suggérer un ensemble d'interventions susceptibles d'augmenter leur contribution au développement socio-économique du monde rural. Dans un premier temps, il sera brièvement fait état de leurs principales caractéristiques et par après, nous suggérerons des éléments d'une politique de développement qui accorderait un rôle spécifique à ces centres de services.

\section{Les centres de services dans le tissu de peuplement québécois}

L'examen de la localisation des centres de services par rapport à l'ensemble du peuplement québécois et surtout en regard de la structure urbaine contribue à illustrer l'importance de leur rôle. C'est en effet en raison de leur localisation qu'ils prennent surtout leur utilité. La plupart d'entre eux desservent des espaces éloignés des villes.

Les agglomérations urbaines de Montréal et Québec concentrent la plupart des villes de plus de 30000 habitants, si bien que la majeure partie de l'espace habité en est à des dizaines voire des centaines de kilomètres de distance. Il n'y a par exemple aucune ville qui atteint cette taille en Gaspésie, en AbitibiTémiscamingue, sur la Côte-Nord et dans la majeure partie des Appalaches et du bouclier laurentien. L'éloignement des villes a des incidences sur les services publics et privés, les structures occupationnelles, les revenus, la démographie, etc. Ainsi, en milieu rural, on observe une relation entre les niveaux de revenus et la localisation et la taille démographique des localités. L'évolution démographique est aussi sensible aux effets de localisation. La propension à la décroissance est plus forte loin des villes et des routes principales. 
À défaut de villes importantes, les petites villes et les centres de services sont les principaux dispensateurs de services pour des centaines de localités et de vastes régions québécoises. Ce sont aussi des centres d'emplois qui polarisent une part significative des travailleurs ruraux et des foyers d'animation qui orientent la vie de relations. Tout en exerçant leurs principales fonctions, celles de fournir emplois et services de base, ils constituent des foyers structurants et des pôles de rétention et de consolidation du peuplement rural.

\section{À défaut de villes importantes, les petites villes et les centres de services sont les principaux dispensateurs de services pour des centaines de locali- tés et de vastes régions québécoises. Ce sont aussi des centres d'emplois qui polarisent une part significative des travailleurs ruraux et des foyers d'animation qui orientent la vie de relations.}

Le qualificatif «centre de services » est réservé dans cette analyse aux municipalités de 2500 à 5000 habitants localisées à l'extérieur des zones périurbaines. Cela correspond à une très grande diversité d'entités territoriales qui se situent dans l'espace de transition entre le rural et l'urbain. Les seuils démographiques choisis correspondent à une zone relativement floue de démarcation dans la fonction services observée lors d'études antérieures ${ }^{1}$ et ne sont qu'une forme de cadrage repère. La démographie ne reflète que bien partiellement l'importance de la fonction services. Le facteur localisation joue un rôle important, si bien qu'en fonction de ce dernier, des localités de moins de 2500 habitants peuvent aussi être de véritables centres de services pour le milieu avoisinant alors que d'autres faisant partie du groupe retenu ont très peu d'influence supralocale. Par ailleurs, des municipalités de plus de 5000 habitants issues d'un processus de fusion et souvent à grande superficie de terrain sont plus facilement assimilables aux centres de services qu'au monde urbain. Il faut donc voir comme préliminaire et indicative la liste de localités retenues ici. Des analyses supplémentaires centrées sur la structure de peuplement et la fonction services s'imposent pour l'établissement d'une politique d'intervention appuyée sur les centres de services.
En 1996, le Québec comptait 168 municipalités de 2500 à 5000 habitants, abritant une population de 595600 personnes. Ces effectifs correspondent à $8,3 \%$ de la population du Québec. Si on soustrait les localités qui font partie des zones périurbaines, le nombre de centres de services s'établit à 116, avec des effectifs totaux de 400934 personnes, représentant $5,6 \%$ de la population québécoise. C'est un chiffre qui semble bien faible, surtout si on considère que les agglomérations et régions métropolitaines de recensement en rassemblent $77,6 \%$. Toutefois, le rôle de ces centres de services ne se mesure pas en regard des agglomérations mais plutôt par rapport au vaste territoire rural qui constitue la majeure partie de l'espace habité.

Ces centres de services se distribuent dans l'espace de manière très inégale à l'intérieur de 58 MRC différentes. Comme toutes les localités du Québec, leur situation actuelle découle d'un long processus évolutif influencé par une multiplicité de variables à caractères géographique, économique, politique, social, administratif, culturel, etc. Leur position dans la structure de peuplement n'a pas été planifiée et n'est que la résultante de toutes les forces qui ont contribué à leur formation pendant de nombreuses décennies. Leur répartition est donc très déséquilibrée, ce qui implique une certaine compétition à certains endroits et des déficits de services ailleurs. Dans certaines régions, ils laissent la place à des villes plus importantes alors qu'ailleurs, les services à caractère régional disponibles sont situés dans des localités de plus faible taille démographique. Mais, où qu'ils soient, ils jouent un rôle essentiel dans la vie rurale, tant par les services qu'ils fournissent que par les emplois qu'ils créent.

La position des centres de services dans la structure de peuplement n'a pas été planifiée et n'est que la résultante de toutes les forces qui ont contribué à leur formation pendant de nombreuses décennies. Leur répartition est donc très déséquilibrée, ce qui implique une certaine compétition à certains endroits et des déficits de services ailleurs.

C'est dans la plaine de Montréal et en Estrie que ces centres de services sont à la fois les plus nombreux et 
les mieux répartis. Ils présentent aussi une distribution assez équilibrée au Saguenay - Lac-Saint-Jean. Partout ailleurs, c'est l'inégale répartition qui prévaut. Ils sont absents sur de vastes espaces, tout particulièrement sur la Côte-Nord, sur la rive nord de la péninsule gaspésienne, dans les Laurentides et Lanaudière. Là où il n'y a pas de ville de taille supérieure, des petites localités rurales les remplacent.

Les centres de services ont en 1996 une population moyenne de 3456 personnes. En fait, le rôle de la taille démographique se manifeste davantage sur le tissu de peuplement et la quantité des services que sur leur nature et leur diversité. En ce qui concerne ces deux paramètres, la localisation géographique, la configuration de la zone à desservir et la nature de l'économie régionale jouent des rôles des plus déterminants.

Pour les résidents des localités concernées de même que pour ceux des zones desservies, la taille démographique est néanmoins importante en raison de ses incidences sur l'infrastructure de services et sur le potentiel de développement. Les municipalités de l'ordre de 5000 personnes ont habituellement une structure économique moins fragile que celles de taille inférieure à 3000 . Cela semble se refléter sur l'évolution démographique.

Entre 1991 et 1996, la population des centres de services a augmenté de 5,4\%, comparativement à 2,3\% pour celle des localités de moins de 2500 habitants, à $1,4 \%$ pour celles de 501 à 1000 habitants, et à 3,5\% pour la population provinciale. Les centres de services ont aussi fait mieux que l'ensemble des six régions métropolitaines de recensement (RMR), dont le taux d'évolution est sensiblement égal à celui du Québec et beaucoup mieux que les 24 agglomérations de recensement, dont la population n'a augmenté que d'un maigre $1,8 \%$.

Affichant des taux d'évolution qui vont de $7,2 \%$ à $8,7 \%$, les trois strates supérieures à 3500 habitants témoignent d'une croissance plutôt exceptionnelle qui n'est dépassée que par la RMR de Hull. Tous ces taux d'évolution sont d'autant plus surprenants qu'ils se manifestent à l'intérieur de l'espace rural. Avec les petites villes, les centres de services sont en partie responsables de l'importante évolution démographique différenciée qui s'y produit.

Affichant des taux d'évolution qui vont de $7,2 \%$ à $8,7 \%$, les trois strates supérieures à 3500 habitants témoignent d'une croissance plutôt exceptionnelle qui n'est dépassée que par la RMR de Hull. Tous ces taux

Tableau 1. Structure occupationnelle par catégorie de localités en 1996

\begin{tabular}{|c|c|c|c|}
\hline \multirow[b]{2}{*}{ Activités } & \multicolumn{3}{|c|}{ Nombre de travailleurs en pourcentage de la main-d'œuvre?2 } \\
\hline & $\begin{array}{l}\text { Localités de } 2500 \\
\text { habitants et moins }\end{array}$ & $\begin{array}{l}\text { Localités de } 2501 \\
\text { à } 5000 \text { habitants }\end{array}$ & Ensemble du Québec \\
\hline Agriculture & 9,0 & 3,9 & 2,2 \\
\hline Pêche et piégeage & 0,5 & 0,5 & 0,1 \\
\hline Exploitation forestière & 2,7 & 1,8 & 0,6 \\
\hline Mines et carrières & 1,1 & 1,8 & 0,5 \\
\hline Primaire & 13,3 & 8,0 & 3,3 \\
\hline Secondaire & 24,3 & 25,9 & 20,9 \\
\hline Tertiaire & 54,1 & 61,8 & 70,9 \\
\hline
\end{tabular}

Source : Statistique Canada, recensement de 1996. 
d'évolution sont d'autant plus surprenants qu'ils se manifestent à l'intérieur de l'espace rural. Avec les petites villes, les centres de services sont en partie responsables de l'importante évolution démographique différenciée qui s'y produit.

La structure occupationnelle des centres de services se différencie à la fois de celle du monde rural et de celle du Québec. Elle affiche certaines caractéristiques du rural tout en s'en démarquant sous plusieurs aspects. Elle comporte une proportion significative de travailleurs agricoles, mais aussi un plus fort pourcentage de travailleurs dans le secteur des services. En fait, elle illustre bien sa spécificité à l'intérieur de la structure de peuplement. Ses différences par rapport aux localités rurales ( 2500 habitants et moins) et au Québec se manifestent dans toutes les catégories d'emplois, mais plus particulièrement dans celles des secteurs primaires et tertiaires (tableau 1). Une proportion identique de travailleurs de la pêche et du piégeage à celle des localités rurales et une plus forte proportion de travailleurs miniers contribuent à illustrer son insertion dans les espaces liés à l'exploitation des ressources. Ces activités minières et halieutiques ne commandent plus une localisation à proximité de la ressource comme l'agriculture et peuvent être associées à différentes catégories de localités et dans bien des cas, à celles qui ont une bonne infrastructure de services.

\section{Politique de développement des centres de services}

Pour favoriser un développement plus équilibré et harmonieux de l'espace québécois, il est impératif d'associer étroitement les centres de services à toute stratégie globale de développement qui impliquerait les agglomérations et l'ensemble du tissu de peuplement urbain et rural. En raison de leur nombre, de leur localisation et de leur signification pour des centaines de milliers de ruraux et de vastes régions économiques, les centres de services méritent une place privilégiée. La prise en compte des seules agglomérations aurait pour effet d'accentuer les distorsions qui prévalent maintenant, compte tenu de leur localisation géographique. Par ailleurs, toute politique de développement régional et rural qui ne tiendrait pas explicitement compte des centres de services serait sérieusement compromise dès le départ. Elle pourrait favoriser la marginalisation des localités les plus éloignées des villes.
Pour favoriser un développement plus équilibré et harmonieux de l'espace québécois, il est impératif d'associer étroitement les centres de services à toute stratégie globale de développement qui impliquerait les agglomérations et l'ensemble du tissu de peuplement urbain et rural. En raison de leur nombre, de leur localisation et de leur signification pour des centaines de milliers de ruraux et de vastes régions économiques, les centres de services méritent une place privilégiée.

Pour maintenir et augmenter sa population, garder la main-d'œuvre qu'il fait instruire et favoriser son développement, le monde rural a besoin de services adéquats et de nouveaux emplois permanents et bien rémunérés dans les secteurs de la transformation et des services. Dans le contexte économique actuel, il est difficile d'imaginer des augmentations significatives d'emplois dans l'exploitation des ressources. Or, quelles que soient les vertus du développement local, il est aussi difficile de concevoir le maintien et la création des services indispensables dans les centaines de petites localités rurales fragiles et souvent excentriques du plateau appalachien et du bouclier laurentien et dans les dizaines de MRC dépourvues de villes sans tenir compte prioritairement des centres de services existants et des localités de taille encore plus petite qui exercent le rôle de dispensateur de services dans leur région respective.

L'évolution démographique des vingt dernières années tend à illustrer la place occupée par les centres de services dans la transformation du monde rural. La plupart ont connu une augmentation de population nettement supérieure à celle de leur région d'appartenance qui, dans bien des cas, fait contraste avec la décroissance continue prévalant à quelques kilomètres de distance. Cette croissance démographique s'est évidemment accompagnée de la création d'emplois, notamment dans le secteur des services. Bon nombre de ces emplois sont aussi occupés par des résidents des localités environnantes. 
Par une politique concertée et à long terme, il y a possibilité de renforcer les rôles structurants et dynamisants des centres de services. Ce faisant, on pourrait contribuer non seulement à favoriser le développement régional, mais aussi à ralentir et idéalement, à diminuer les déséquilibres qui se manifestent dans la structure de peuplement du Québec. Ces déséquilibres sont nuisibles tant sur le plan social qu'économique. Ils créent des problèmes de services tout autant dans les espaces à forte densité de population que dans ceux à trop faible densité et occasionnent des coûts d'implantation et de restructuration à des endroits alors qu'il y a gaspillage par sousutilisation ailleurs.

Par une politique concertée et à long terme, il y a possibilité de renforcer les rôles structurants et dynamisants des centres de services. Ce faisant, on pourrait contribuer non seulement à favoriser le développement régional, mais aussi à ralentir et idéalement, à diminuer les déséquilibres qui se manifestent dans la structure de peuplement du Québec.

Une stratégie de développement fortement axée sur les centres de services devrait idéalement impliquer les quatre niveaux de gouvernement, c'est-à-dire le fédéral, le provincial, les MRC et les municipalités. Les CRCD (conseils régionaux de concertation et de développement) sont aussi concernés avec leur planification stratégique, de même que les CLD (centres locaux de développement) et les SADC (sociétés d'aide au développement des collectivités). Le rôle des municipalités devrait s'inscrire dans des orientations de longue durée établies dans les instances gouvernementales supérieures et inscrites notamment dans les schémas d'aménagement et plans d'urbanisme.

Dans un premier temps, le gouvernement du Québec devrait procéder à l'identification des municipalités devant faire partie du réseau des centres de services et leur donner un statut officiel. Ces localités devraient aussi être reconnues par le gouvernement fédéral au même titre que par le gouvernement québécois comme territoires d'interventions particulières en vue de renforcer l'armature du tissu de peuplement. Cette reconnaissance devrait ultérieurement avoir ses implications sur toutes les politiques et interventions sectorielles des ministères et organismes d'État et contribuer à influencer à long terme la déconcentration administrative et la localisation de services de première nécessité et dans certains cas, de portée régionale. Cette reconnaissance officielle est l'élément le plus fondamental, si elle est accompagnée d'une véritable volonté politique. Ses effets ne devraient pas être qu'administratifs et économiques ; ils devraient aussi avoir une dimension symbolique aux retombées perceptuelles et idéologiques non négligeables.

Les interventions devraient s'appliquer simultanément sur au moins trois plans, soit le renforcement de l'infrastructure de services publics, la création d'emplois et la qualité de l'aménagement. Ce sont trois éléments qui évoluent en interrelation constante et qui doivent être considérés de façon intégrée. Les emplois génèrent des services, mais pour être créés, les emplois exigent aussi un environnement adéquat. Des services de qualité, d'accessibilité facile et un bon cadre de vie sont par ailleurs indispensables pour favoriser le maintien et l'augmentation des populations.

Le gouvernement du Québec devrait procéder à l'identification des municipalités devant faire partie du réseau des centres de services et leur donner un statut officiel. Ces localités devraient aussi être reconnues par le gouvernement fédéral au même titre que par le gouvernement québécois comme territoires d'interventions particulières en vue de renforcer l'armature du tissu de peuplement.

La question des services publics ou gouvernementaux doit être envisagée sur un double plan, soit la localisation et la nécessité de fournir des services adéquats. Le choix des lieux d'implantation de services de portée supralocale ne doit pas obéir aux seules planifications et orientations des organismes responsables et aux influences politiques, mais s'inscrire dans la stratégie globale de consolidation de l'armature du tissu de peuplement. Par des localisations pertinentes, en évitant une inutile dispersion, on peut contribuer simultanément à en favoriser l'accessibilité à la population et à consolider des structures économiques et centres d'emplois. 
L'implantation des services publics doit être envisagée dans une perspective d'amélioration de la qualité de vie des résidents et du potentiel de développement des espaces concernés. Dans certains cas, ça peut impliquer une déconcentration accrue de l'appareil gouvernemental et des adaptations normatives aux spécificités régionales. Mais aussi, à bien des endroits, il faudrait passer d'une politique de maintien du minimum et même de déstructuration à des objectifs de consolidation, de renforcement et d'atténuation des disparités provinciales.

Les interventions devraient s'appliquer simultanément sur au moins trois plans, soit le renforcement de l'infrastructure de services publics, la création d'emplois et la qualité de l'aménagement. Ce sont trois éléments qui évoluent en interrelation constante et qui doivent être considérés de façon intégrée.

La dimension «aménagement » concerne toutes les instances gouvernementales en raison de ses implications sur les infrastructures, la gestion des ressources et de l'environnement et le contrôle de toutes les formes d'utilisation du sol. Le renforcement des centres de services désignés devra faire partie des orientations des schémas d'aménagement. Dans certaines MRC, cela pourra exiger un effort particulier d'articulation avec des objectifs de renforcement de villes de taille supérieure. La composante esthétique et qualitative de l'aménagement devra aussi mériter une attention particulière, notamment sur le plan de l'urbanisme et de la réglementation municipale.

Au plan de la création d'emplois, les centres de services devraient être considérés comme zones spéciales. À ce titre, ils devraient bénéficier de mesures particulières et suffisamment fortes pour compenser des inconvénients de localisation. Comme il s'agit de
Le choix des lieux d'implantation de services de portée supralocale ne doit pas obéir aux seules planifications et orientations des organismes responsables et aux influences politiques, mais s'inscrire dans la stratégie globale de consolidation de l'armature du tissu de peuplement. Par des localisations pertinentes, en évitant une inutile dispersion, on peut contribuer simultanément à en favoriser l'accessibilité à la population et à consolider des structures économiques et centres d'emplois.

consolider des centres de services et même de faire émerger des villes, il faut mettre en place des éléments d'attraction capables de faire compétition aux villes à meilleur potentiel. La gamme des entreprises visées devrait être large et surtout, ne pas être limitée au seul secteur de transformation des ressources locales et régionales ou encore à des entreprises à faibles composantes technologiques et à bas salaires. Il faut aussi des entreprises aptes à favoriser la rétention de la main-d'œuvre spécialisée issue de la région. Les emplois créés doivent contribuer à l'augmentation des niveaux de revenus régionaux et par voie de relation, à l'amélioration des services du secteur privé et de la qualité de vie.

Notes et références

1 DUGAS, Clermont (1984). La ruralité québécoise : évolution et perspectives, Statistique Canada, Division de la recherche et de l'analyse, document no 6, 58 p. ; DUGAS, Clermont (1999). Le monde rural québécois et ses centres de services : dynamisme, marginalité, marginalisation, Chaire Desjardins en développement des petites collectivités, Solidarité rurale du Québec et ministère des Régions, 96 p.

2 La catégorie « travailleurs non classifiés » n'a pas été prise en compte pour le calcul des pourcentages. 